\title{
Propagation velocity of epileptiform activity in the hippocampus
}

\author{
J. Holsheimer ${ }^{1}$ and F.H. Lopes da Silva ${ }^{2}$ \\ ${ }^{1}$ Department of Electrical Engineering, University of Twente, P.O. Box 217, NL-7500 AE Enschede, The Netherlands \\ ${ }^{2}$ Department of Experimental Zoology, University of Amsterdam, Kruislaan 320, NL-1098 SM Amsterdam, The Netherlands
}

Summary. The propagation of epileptiform burst activity was investigated in the CA1 area of the in-vitro hippocampal slice preparation of the guinea pig. This activity was provoked by $0.1 \mathrm{mM}$ 4-aminopyridine in the bathing medium and was recorded in the pyramidal layer with an array of eight electrodes. The delay between the first population spike of a burst recorded with different electrodes was calculated using the cross-correlation function. The propagation velocity was estimated from the delays and the electrode intervals. It was found that the velocity of spontaneous and evoked epileptiform bursts varies between 0.15 and $5 \mathrm{~m} / \mathrm{s}$ and is not confined to the range of conduction velocities of the fibre systems in CA1 $(0.3-0.55$ and $1.0-1.8 \mathrm{~m} / \mathrm{s}$ ). Different velocities can be present in different parts of the CA1 area and the initiation of spontaneous bursts is not confined to the CA2-3 areas, but can also occur in CA1. Burst activity also propagated in a low calcium-high magnesium medium. Different mechanisms of propagation are discussed and it is argued that the propagation velocity due to ephaptic interaction may vary largely. It is concluded that epileptiform activity can be propagated not only by synaptic connections at or near the pyramidal layer, but also by way of electrical field effects of population spikes.

Key words: Hippocampus - Epileptiform burst Propagation velocity - Nerve fibres - Ephaptic interaction

\section{Introduction}

In the generation of epileptic activity mechanisms both at the cellular and at the population level play an important role. Although the investigation of

Offprint requests to: J. Holsheimer (address see above) epileptic activity has long been concentrated on cellular properties (Prince 1978), the questions of how synchronization and propagation of this activity in large neuronal populations take place, are receiving increased attention. Both aspects have been investigated in the in-vitro hippocampal slice preparation. The hippocampus is appropriate for the investigation of population properties, since it has a relatively simple laminar arrangement of its neurons. Evoked potentials and epileptiform activity are associated with the almost simultaneous discharge of a large number of these neurons and give rise to field potentials with large amplitudes (Andersen et al. 1971; Schwartzkroin and Prince 1978).

The site of generation of epileptiform bursts in the hippocampus has been investigated by several authors (Schwartzkroin and Prince 1978; Wong and Traub 1983; Voskuyl and Albus 1985; Mody et al. 1987). For different models of epilepsy (penicillin, bicucullin, 4-aminopyridine, low magnesium) it was found that burst activity was initiated in the CA2-3 area and propagated towards the CA1 area. Voskuyl and Albus (1985) found a propagation from CA3 to CA1 at $0.1-0.5 \mathrm{~m} / \mathrm{s}$ for one type and $0.01 \mathrm{~m} / \mathrm{s}$ for a second type of spontaneous 4-AP bursts. Schwartzkroin and Prince (1978) reported a velocity of approximately $0.1 \mathrm{~m} / \mathrm{s}$ in the penicillin model and Knowles et al. (1987) found in CA2-3 an average value of $0.13 \mathrm{~m} / \mathrm{s}$ in the picrotoxin model. In the low calcium model of epilepsy a very slow spread was observed in CA1, at a mean velocity of $0.00174 \mathrm{~m} / \mathrm{s}$ (Konnerth et al. 1986).

Several mechanisms may be involved in the propagation of epileptic activity in the hippocampus and other structures. The role of chemical synaptic transmission has been considered essential for the spread of epileptiform activity (Dichter and 
Spencer 1969; Ayala et al. 1973; Schwartzkroin and Prince 1978; Prince 1978; Gjerstad et al. 1981; Johnston and Brown 1981). However, seizure-like discharges have been observed in hippocampal slices in which chemical transmission was blocked by a low calcium-high magnesium (or manganese) solution (Taylor and Dudek 1982a; Jefferys and Haas 1982; Yaari et al. 1983; Snow and Dudek 1984a; Richardson et al. 1984; Konnerth et al. 1986). It was concluded by Taylor and Dudek (1982a, 1984a, b), Haas and Jefferys (1984), Richardson et al. (1984), Snow and Dudek (1984b) and Yim et al. (1986) that electrical field effects (ephaptic interaction) may contribute to the recruitment and synchronization of the population of CA1 pyramidal cells by depolarizing marginally active neurons. The modelling studies of Traub et al. $(1985 \mathrm{a}, \mathrm{b})$ led to the same conclusion. A similar mechanism of propagation of hippocampal seizures was proposed previously by Green and Petsche (1961), who pointed out that synchronized activity could easily occur due to transient electrical fields evoked by simultaneously generated action potentials (population spikes). Jefferys (1981) demonstrated that externally applied current pulses giving rise to field potential gradients of 5$10 \mathrm{mV} / \mathrm{mm}$ could modify the excitability of dentate granule cells, while population spikes in the hippocampus may generate potential gradients as high as $50 \mathrm{mV} / \mathrm{mm}$ along the somadendritic axis.

Electrotonic or gap junctions are observed in several hippocampal areas (Schmalbruch and Jahnsen 1981; MacVicar et al. 1982; Taylor and Dudek 1982b). Since electrotonic depolarizations were only found in a small percentage of CA1 pyramidal cells and dye injection usually stained only $2-3$ cells, this type of interaction is not considered to be a probable mechanism of propagation in the CA1 area (Haas and Jefferys 1984; Richardson et al. 1984).

During epileptic activity extracellular ionic concentrations may change significantly. The increase of $\mathrm{K}^{+}$(Fisher et al. 1976; Heinemann et al. 1977; Prince 1978; Krnjevič et al. 1982; Somjen and Giacchino 1985) may depolarize neuronal membranes by $10 \mathrm{mV}$ or more (Haas and Jefferys 1984) and thus increase their excitability. Therefore, changes in the ionic environment may facilitate synchronization of neuronal activity. In the low calcium model it may also account for the very slowly propagating seizure-like events (Yaari et al. 1986)

Different spreading mechanisms of epileptic activity may be characterized by various propagation velocities. In order to obtain a better understanding of such mechanisms we determined the propagation velocity of epileptiform bursts in hippocampal in-vitro slices, using the 4-aminopyridine epilepsy model in which this activity is confined to the CA1-3 areas (Fricke and Prince 1984; Voskuyl and Albus 1985). Multielectrode recordings were made in the pyramidal layer of the CAl area, and propagation velocities of spontaneous epileptiform bursts were compared with the values of evoked bursts and the velocities of the fibre systems in CA1.

\section{Methods}

\section{Experimental techniques}

Twelve guinea pigs $(450-600 \mathrm{~g})$ were anaesthetized with ether, decapitated and the brains quickly removed. The hippocampi were isolated and slices $(0.6 \mathrm{~mm}$ thick $)$ were cut with a set of 5 razor blades in a plane parallel to the lamellar structure. The slices were preincubated in a bathing medium at room temperature, consisting of (in mM) NaCl 124, $\mathrm{KCl} 5$ or $6, \mathrm{NaH}_{2} \mathrm{PO}_{4}$ 1.24, $\mathrm{MgSO}_{4} 1.3, \mathrm{NaHCO}_{3} 26, \mathrm{CaCl}_{2} 2$ and glucose 10 . The medium was saturated with $95 \% \mathrm{O}_{2}$ and $5 \% \mathrm{CO}_{2}$. After 1 hour slices were transferred to a perfusion chamber with the same bathing medium, held at a temperature of $32.0-32.5^{\circ} \mathrm{C}$. The chamber $(2.7 \mathrm{ml})$ was perfused at a velocity of $1 \mathrm{ml} / \mathrm{min}$. Humified gas $\left(95 \% \mathrm{O}_{2}-5 \% \mathrm{CO}_{2}\right)$ was blown over the slices, which were on a nylon gauze just underneath the surface of the bathing medium. The condition of the slices, as shown by the electrophysiological recordings, could be kept constant for 46 hours in this perfusion chamber. In some experiments different concentrations of $\mathrm{KCl}$ and $\mathrm{CaCl}_{2}$ were used without correcting for the change in osmolarity. In experiments with low $\mathrm{Ca}^{2+}$ $(0.2 \mathrm{mM})$ the $\mathrm{CaCl}_{2}$ was replaced by $\mathrm{MgCl}_{2}$.

Field potentials were recorded simultaneously with an eight-electrode array developed in our laboratory (Verloop and Holsheimer 1984). The array, with electrode diameters of $33 \mu \mathrm{m}$ and tip intervals of 0.1 or $0.2 \mathrm{~mm}$, was positioned by a micromanipulator in the pyramidal layer of the relatively flat area $\mathrm{CAlb}$ (Lorente de Nó 1934). The reference electrode was an $\mathrm{Ag}-\mathrm{AgCl}$ electrode placed at the bottom of the perfusion chamber $(3 \mathrm{~mm}$ underneath the slices). The recording electrodes were connected to eight pre-amplifiers $\left(10^{15} \mathrm{Ohm} \| 0,6 \mathrm{pF}\right)$. The amplification systems (bandwidth $0.5-5000 \mathrm{~Hz}$ ) fed the field potentials into a 14-channel FM recorder (Ampex FR 1300). Stimulus artefacts were suppressed by a stimulus-triggered hold-pulse $(0.6 \mathrm{~ms})$, fed into a sample-and-hold amplifier in each amplification system. This pulse was also fed into the analog recorder.

An isolated current source was used for stimulation. A small cathodal electrode was positioned in the slice by a micromanipulator and a larger anodal one was located at the bottom of the perfusion chamber. The current source was driven by an Apple II microprocessor. Current pulses could be generated at 15 discrete values ranging from $0.02-0.30 \mathrm{~mA}$, with a duration varying from $0.02-0.20 \mathrm{~ms}$ and variable pulse intervals. Usually intervals of $5 \mathrm{~s}$, a pulse duration of 0.05 or $0.1 \mathrm{~ms}$ and a current of $0.1-0.2 \mathrm{~mA}$ were used.

Epileptiform activity was provoked by adding $0.05-$ $0.10 \mathrm{mM}$ 4-aminopyridine (4-AP) to the bathing medium.

\section{Estimation of time delay}

During the experiments the recorded signals were monitored on a dual channel storage oscilloscope. Delays were estimated from 


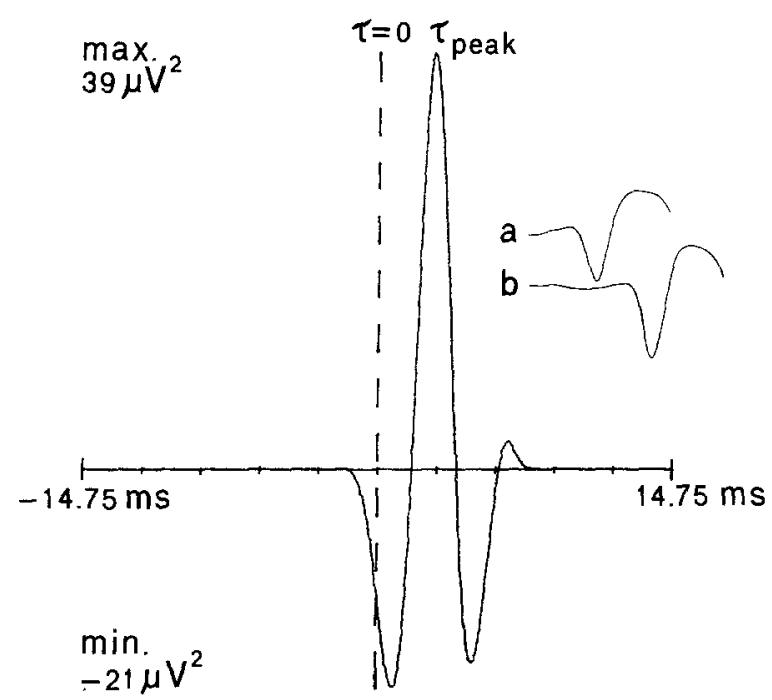

Fig. 1. Cross-correlation function of 2 PSs ( $a$ and $b$ ) used for the estimation of their time interval (resolution $=57.55 \mu \mathrm{s}$ ); in this example the delay between $\tau_{\text {peak }}$ and $\tau=0$ is $3.165 \mathrm{~ms}$

the intervals between the peaks of the corresponding population spikes (PSs), recorded with different electrodes. In control experiments delays between compound action potentials (CAPs), recorded from a stimulated fibre bundle, were estimated in the same way.

Field potentials stored on tape were sampled at a rate of $2000 / \mathrm{s}$ or $2172 / \mathrm{s}$ by a PDP $11 / 40$ computer. (Previous frequency analysis of the signals to be analysed (PSs) showed that the power above $800 \mathrm{~Hz}$ was negligible.) Sampling of an evoked burst was started by the preceding stimulus pulse. In order to increase the signal-to-noise ratio the average value of 4-6 responses was calculated for each channel and stored on disk. This could be done since bursts evoked in the same way were similar, except when an evoked burst was preceded by a spontaneous one within $0.1 \mathrm{~s}$. The sampling of a spontaneous burst was triggered by the negative-going slope of the first PS from the channel which showed the earliest response. In contrast to the responses elicited by electrical stimulation the shapes of the spontaneous bursts were not always similar. In these cases we stored single bursts instead of averages.

We defined the delay of a propagating epileptiform burst, recorded simultaneously by two electrodes, as the delay between the first PSs of the two recordings. The delay was estimated by calculating the position of the peak in the cross-correlation function of the two PSs. For each channel the first PS was selected by a tapered data window of appropriate length. In order to increase the resolution of the delay the resolution of the cross-correlation function was increased eight times by an interpolation procedure (van der Vliet et al. 1980). An example of the interpolated cross-correlation function is shown in Fig. 1. The calculated delays were corrected for differences in delay between the channels, introduced by the analog recorder and the A-D converter. Delays $\Delta t$ of each (average) burst were computed between the PS at one of the extreme electrodes and those at all other electrodes. A plot was made of the values of $\Delta t$ against the distance $\Delta x$ between the electrodes (measured under a microscope). If we assume a constant propagation velocity, this can be estimated by the best-fit line relating to $\Delta x$ and $\Delta t$.

\section{Results}

Within a few minutes after the application of 4-AP, epileptiform bursts occurred regularly every $2-3 \mathrm{~s}$ in the CA1-2 areas, but not in the dentate area (cf. Fricke and Prince 1984; Voskuyl and Albus 1985). These bursts could also be triggered by a weak stimulus in one of the CA1-3 areas. When the bursts were triggered the direction of propagation was dependent on the stimulation site. If a stimulus was given at the subicular side of the electrode array in CA1, the response propagated from the subicular towards the CA3 side and vice versa.

The experiments were done on 22 hippocampal slices: 13 for measurements of the propagation velocity of 4-AP induced epileptiform activity, 7 for measurements of the propagation velocities of the fibre systems in CA1 in a bathing medium without 4-AP and 2 for both.

\section{Propagation velocities of fibres in $C A I$}

In the control experiments different fibre bundles in CA1 were stimulated and recordings were usually made with the electrode array in the same layer as the stimulating electrode (Fig. $2 \mathrm{~A}$ ). This electrode was placed in CAl at one side of the recording electrode array and the Schaffer collaterals (in the middle of the stratum radiatum), the alvear fibres and fibres in the stratum oriens and the stratum radiatum close to the pyramidal layer (commissural and other fibres) were stimulated. Delays between the CAPs of a fibre bundle or the PSs of the pyramidal somata were estimated from the display on the storage oscilloscope and also by means of the cross-correlation method. When both CAPs and PSs were present in the same recording, the time interval between CAP and PS sometimes increased a little at increasing distance from the stimulating electrode (Fig. 2A, 2). Results are shown in Fig. $2 \mathrm{~B}$. The propagation velocities, calculated from the delays, are summarized in Fig. $4 \mathrm{~A}$. The mean velocity of the Schaffer collaterals was $0.34 \mathrm{~m} / \mathrm{s}$ (s.d. $=0.06, n=8$ ), of stratum oriens and commissural fibres $0.48 \mathrm{~m} / \mathrm{s}$ (s.d. $=0.04, n=4)$ and alvear fibres had a mean value of $1.32 \mathrm{~m} / \mathrm{s}$ (s.d. $=0.35, n=6$ ).

\section{Propagation of epileptiform bursts}

After the application of 4-AP epileptiform bursts were recorded in the pyramidal layer. Spontaneous and evoked bursts had similar shapes. This similarity was true for the complete laminar profiles (see Discussion). Therefore propagation velocities of 

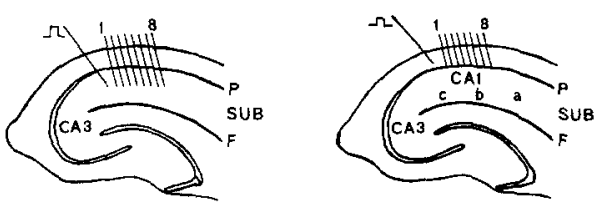

A

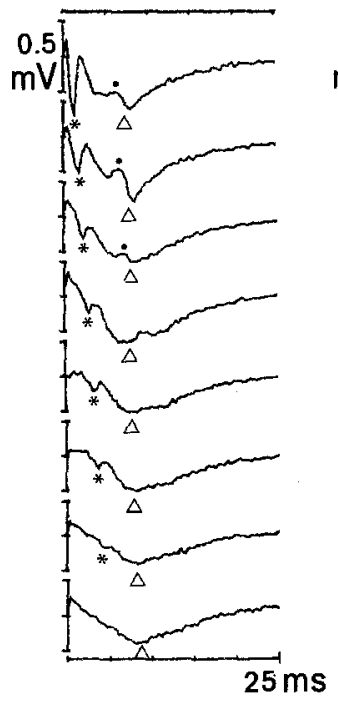

1

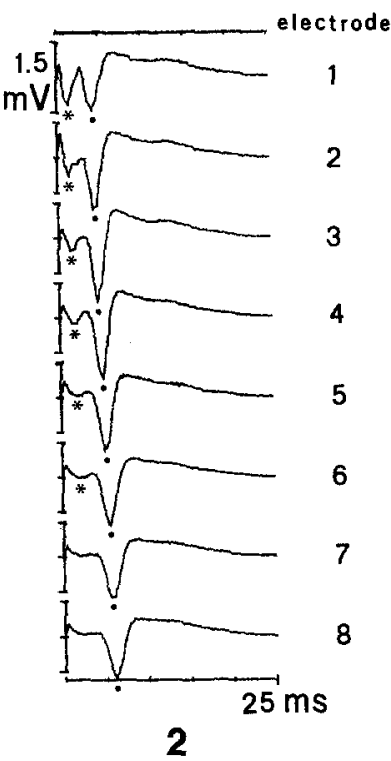

SUB

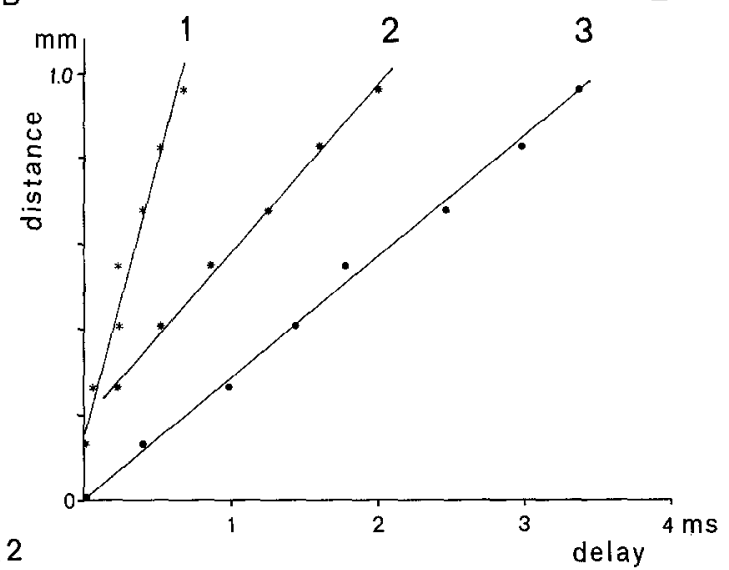

CA 2

delay

Fig. 2A, B. Control experiments. A Averaged evoked field potentials at stimulation of Schaffer collaterals (1) and stratum oriens fibres (2), recorded in the hippocampal area CA1 b with 8 electrodes at intervals of $\approx 0.13 \mathrm{~mm}$ in the stratum radiatum (1) and at the border of stratum oriens/pyramidale (2); trace 1 at the CA3-side of the recording area, positive up; CAPs indicated by an asterix, field EPSPs by a triangle and PSs by a dot; P: pyramidal layer, F : hippocampal fissure, SUB: subiculum. B Plot of electrode distance versus delay; the propagation velocity of the fibre systems is shown by the slope of the lines; 1. alvear fibres $(1.3 \mathrm{~m} / \mathrm{s}), 2$. stratum oriens fibres $(0.39 \mathrm{~m} /$ $\mathrm{s})$, 3. Schaffer collaterals $(0.28 \mathrm{~m} / \mathrm{s})$; propagation towards the subiculum

spontaneous burst activity were compared with velocities of bursts triggered by stimulation of specific pathways. In order to evoke bursts, stimuli were given in one of the CA1 layers or in the pyramidal layer of CA2-3. The threshold current for triggering a burst was less than $50 \%$ of the threshold current required to evoke a Schaffer collateral response before the application of 4-AP. The first PS of bursts triggered by stimulation had a mean amplitude of $2.8 \mathrm{mV}$ (s.d. $=1.3 \mathrm{mV}, n=9$ ). For spontaneous bursts the mean was $1.5 \mathrm{mV}$ (s.d. $=0.8 \mathrm{mV}, n=5$ ). No relationship was found between the PS amplitude and the propagation velocity. Successive stimuli at the same site resulted in almost identical latencies at each electrode. Differences in delay were not more than once (sometimes twice) the resolution of the interpolated cross-correlation function $(58 \mu \mathrm{s})$. Responses with different shapes and latencies, occurring if a stimulus was given less than $0.1 \mathrm{~s}$ after a spontaneous burst, were not used. Successive spontaneous bursts also had almost identical delays between electrodes. The delays calculated from the recordings shown in Fig. $3 \mathrm{~A}$ are presented as a function of electrode distance in Fig. $3 \mathrm{~B}$. Note that curve 1 propagates towards the CA3-side, due to stimulation at the subicular side.

The propagation velocities calculated from all recordings of evoked and spontaneous epileptiform bursts are summarized in Fig. 4 B. In this histogram the range of propagation velocities is divided into 3 intervals: $0.15-0.55$ (I), $0.55-1.8$ (II) and $>1.8 \mathrm{~m} /$ $\mathrm{s}$ (III). Interval I includes the range of the Schaffer collaterals, the commissural and stratum oriens fibres and interval II includes the range of the alvear fibres. The propagation velocities found for these fibre systems are shown in Fig. 4 A. No simple relationship was found between the stimulus condition and the propagation velocity of epileptiform activity. The propagation of spontaneous bursts mostly had a velocity in interval I $(n=10)$ or II $(n=10)$, and some in interval III $(n=3)$. See Fig. 5 (curve 1). With stimulation of the alveus the velocities were almost evenly distributed over the three ranges $(n=6,5,5)$. When stimulating the CA2-3 pyramidal layer only half of the results corresponded with the velocity of Schaffer collateral, stratum oriens and commissural fibres $(n=9,5,4)$. In one slice the propagation velocity of the PSs at alvear stimulation was estimated before and after the application of 4-AP. The values were the same $(1.6 \mathrm{~m} / \mathrm{s})$, but spontaneous 4-AP bursts had a higher velocity $(3.5 \mathrm{~m} / \mathrm{s})$. It can be concluded that velocities above $1.8 \mathrm{~m} / \mathrm{s}$ occur with stimulation of the alveus in CA1 or the pyramidal layer in CA2-3 and with spontaneous bursts, but not with stimulation of the other fibre systems in CA1. Another conclusion is that the velocities we found between 0.55 and $1.0 \mathrm{~m} / \mathrm{s} \quad(n=10)$ and higher than $1.8 \mathrm{~m} / \mathrm{s}$ $(n=12)$ do not fit those of the fibre systems in CA1 under control conditions. 


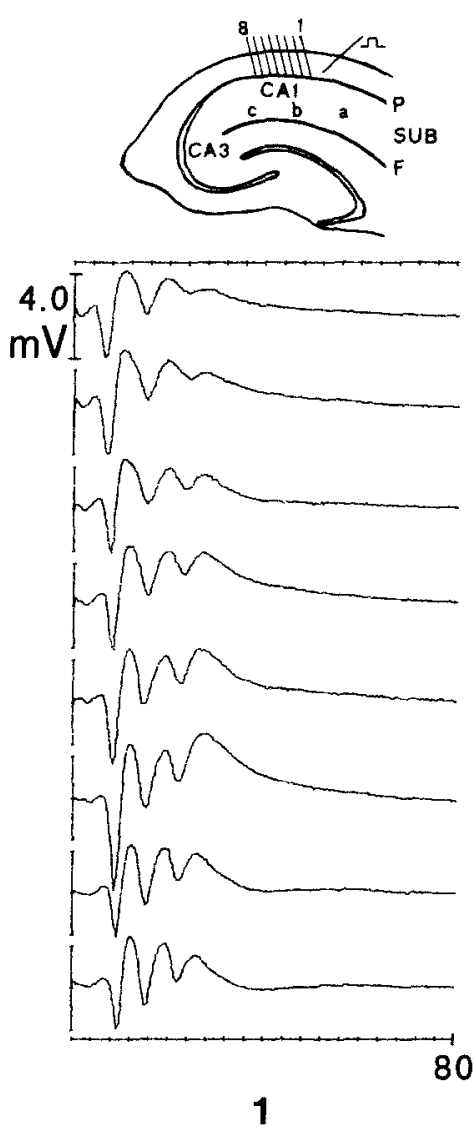

1
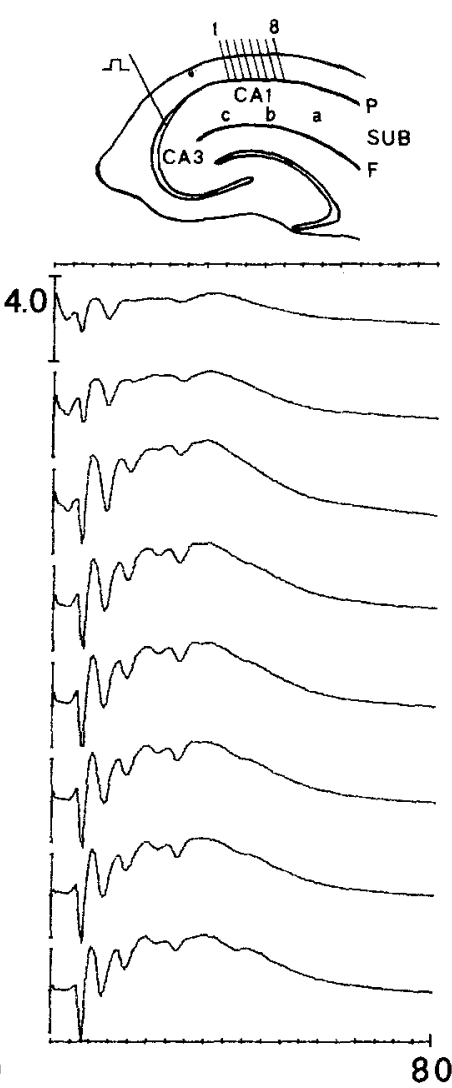

2

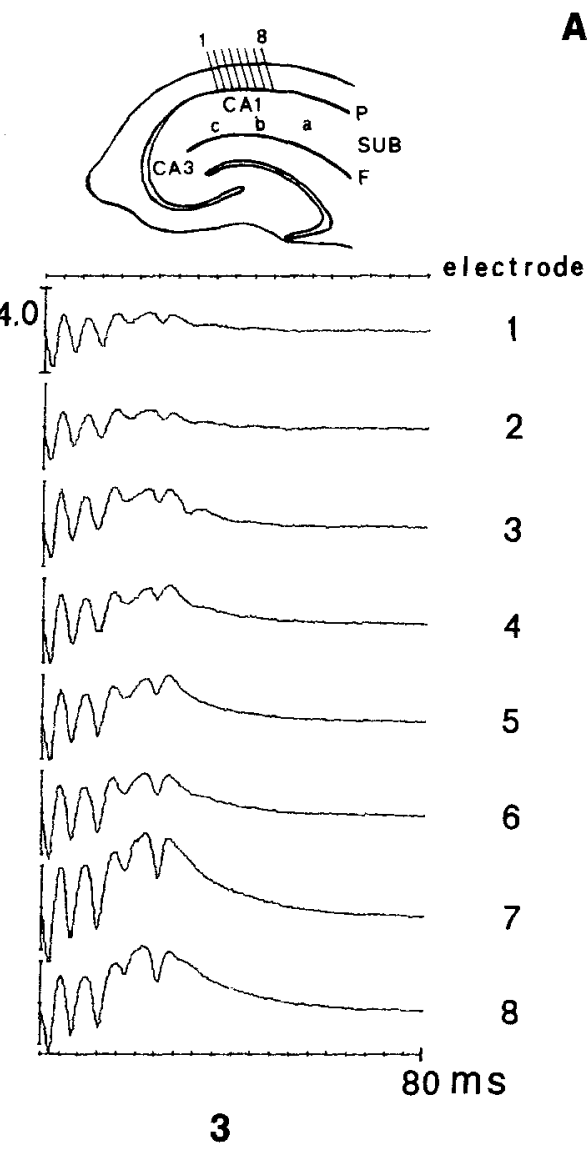

SUB

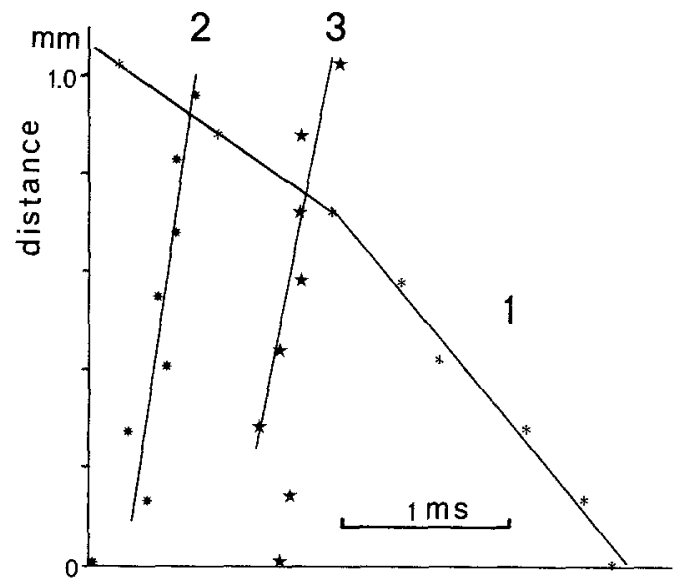

CA2

delay

Spontaneous bursts were recorded and analysed from 9 slices. In 4 slices they originated from the recording area in the central part of CA1 and propagated in two directions. Results are shown in Fig. 5 (curves 2 and 3). In the other 5 slices the burst activity came either from the CA2side of the recording area $(n=3)$ or from the subicular side $(n=2)$. The finding that spontaneous bursts can develop in the CA1 area was confirmed
Fig. 3. A Averaged epileptiform bursts recorded simultaneously in the CA1 b pyramidal layer with 8 electrodes at intervals of $\approx 0.13 \mathrm{~mm} ; 1$. response to stimulation of stratum oriens in area $\mathrm{CA} 1 \mathrm{a}, 2$. response to stimulation of the pyramidal layer in CA3, 3. spontaneous bursts; trace 1 at the subicular side (1) or at the CA3-side (2 and 3 ) of the recording area, positive up. B Plot of electrode distance versus delay between the first PS of the recordings shown in A; propagation velocities: 0.25 and $0.45 \mathrm{~m} / \mathrm{s}(1), 2.5 \mathrm{~m} / \mathrm{s}(2)$ and $1.9 \mathrm{~m} / \mathrm{s}(3)$

by experiments in which incisions were made in the slice. In these cases, even when the CA1 area was completely isolated from the other parts of the hippocampal slice, regular spontaneous burst activity could occur in this area.

In several slices the propagation velocity was not the same in different parts of the CAlb area. This occurred with spontaneous bursts (4 slices) as well as with bursts evoked with stimulation in CA2-3 (5 slices) and in the alveus/oriens of CA1 (3 slices). An increase in velocity (Fig. $3 \mathrm{~B}$, curve 1 and Fig. 5, curve 4) was found as well as a decrease (Fig. 5 , curve 5), by a factor ranging from 2 to 6 . This phenomenon was independent of the direction of propagation in CAl and a shift in propagation velocity does not seem to be confined to a specific site in the CAlb area. 

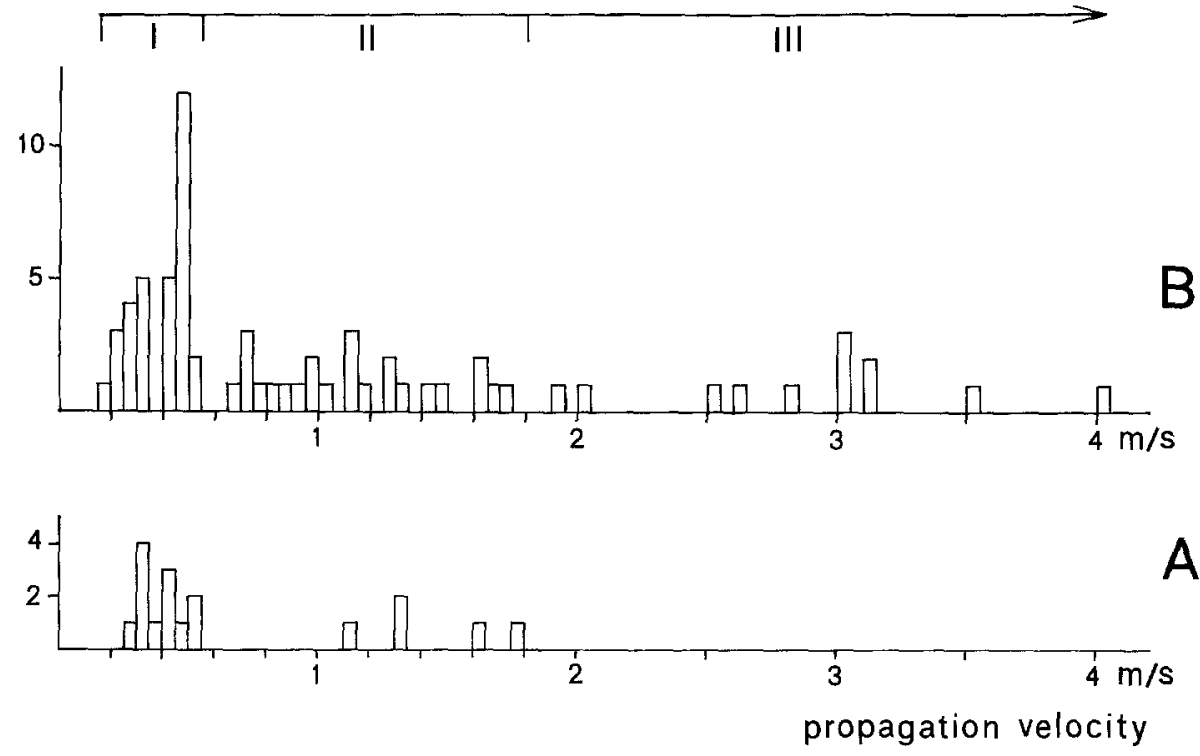

SUB

$\mathrm{CA} 2$

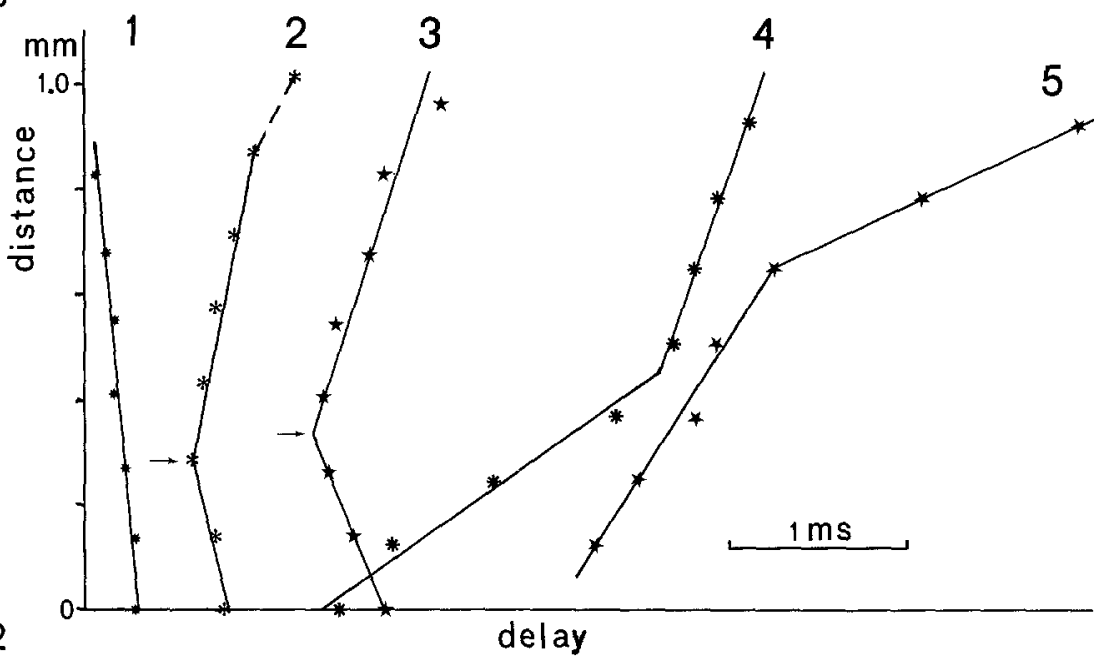

Fig. 4 A. B. Distribution of the propagation velocity in the hippocampal CA1 area: A fibre bundles $(n=17)$ in control experiments, $\mathbf{B}$ epileptiform bursts $(n=68)$, subdivided into velocities of $0.15-0.55 \mathrm{~m} / \mathrm{s}(\mathrm{I}), 0.55-1.8 \mathrm{~m} / \mathrm{s}$

(II) and $>1.8 \mathrm{~m} / \mathrm{s}$ (III)

Fig. 5. Plots of electrode distance versus delay. 1. spontaneous epileptiform bursts propagated from the subicular to the CA2 side at a velocity of $3.6 \mathrm{~m} / \mathrm{s}$; 2. spontaneous bursts initiated in the recording area in CA1b (see arrow) and propagated towards the subiculum at a velocity of $1.7 \mathrm{~m} / \mathrm{s}$ and at $1.4 \mathrm{~m} / \mathrm{s}$ towards CA2; 3. spontaneous bursts propagated towards the subiculum at a velocity of $1.1 \mathrm{~m} / \mathrm{s}$ and at $0.8 \mathrm{~m} / \mathrm{s}$ towards CA2; 4-5. spontaneous epileptiform bursts with different propagation velocities in different parts of the area CA1 b: 4 . velocity of $0.24 \mathrm{~m} / \mathrm{s}$ increased to $1.0 \mathrm{~m} / \mathrm{s}, 5$. velocity of $0.55 \mathrm{~m} / \mathrm{s}$ decreased to $0.17 \mathrm{~m} / \mathrm{s}$; propagation from the $\mathrm{CA} 2$-side to the subicular (SUB) side

\section{Propagation velocity and ionic concentrations}

Because the extracellular concentration of $\mathrm{K}^{+}$in the hippocampus increases during epileptiform activity (Fisher et al. 1976), we were interested in the effect of an increase of the $\mathrm{K}^{+}$concentration in the bathing medium on the propagation of epileptiform bursts. $\mathrm{K}^{+}$concentrations of 3,5 and $8 \mathrm{mM}$ were applied to 2 slices, but we did not observe any significant change in the propagation velocity of the bursts. However, an increase of $\mathrm{K}^{+}$in the bathing medium from $3-5$ to $8-9 \mathrm{mM}$ led to an increase of the amplitude of the first PS varying from $30 \%$ to more than $100 \%$.

We also investigated the effect of low extracellular $\mathrm{Ca}^{2+}$ on the propagation of epileptiform and non-epileptiform activity. After testing the slices in a normal medium with or without 4-AP, they were incubated in a medium with $0.2 \mathrm{mM} \mathrm{Ca}^{2+}$ and $3.3 \mathrm{mM} \mathrm{Mg}^{2+}$ for two hours. Because the amplitude of the PS was reduced to less than $1 \mathrm{mV}$ in this medium, we increased the $\mathrm{K}^{+}$concentration to $6.5-7.0 \mathrm{mM}$ in order to obtain a larger signal. In this medium, with or without 4-AP, we observed propagation of PSs after stimulation of the alveus at velocities varying from $1-5 \mathrm{~m} / \mathrm{s}$. This range not only includes the normal propagation velocity of alvear fibres, but also higher velocities. These experiments confirm the results published by several authors, that in slices with low $\mathrm{Ca}^{2+}$ and high $\mathrm{Mg}^{2+}$ (or $\mathrm{Mn}^{2+}$ ), when synaptic transmission is blocked, interaction between neurons is still possible (see Introduction). 


\section{Discussion}

The shapes of spontaneous and electrically triggered 4-AP bursts were found to be similar, as were the range of propagation velocities of spontaneous bursts and bursts triggered by stimulation of the CA3 pyramidal layer or the alveus in CA1. Therefore the propagation of these types of bursts may share the same mechanisms. The wide range of velocities found $(0.15-5 \mathrm{~m} / \mathrm{s})$ indicates that different mechanisms of propagation may be involved. One way is the propagation by a local fibre system in CA1. The velocities found for these fibres are in good agreement with data from literature for the guinea pig and the rat (Andersen et al. 1978; Leung 1979; Tielen et al. 1981). It was shown (Fig. 4) that part of the bursts propagate at a velocity within this range $(0.3-0.55 \mathrm{~m} / \mathrm{s}$ and $1.0-1.8 \mathrm{~m} / \mathrm{s}$ ). However, these velocities may differ somewhat in the epileptogenic slice where the extracellular $\mathrm{K}^{+}$concentration is increased (Heinemann et al. 1977; Somjen and Giacchino 1985; Heinemann 1987). Poolos et al. (1987) reported a decrease in propagation velocity of fibres in the stratum radiatum of $\mathrm{CA} 1$ by $\approx 30 \%$ at an increase of the interstitial $\mathrm{K}^{+}$concentration from 3 to $9 \mathrm{mM}$. If there is any effect of extracellular $\mathrm{K}^{+}$on the propagation velocity of fibres in the epileptogenic slice, it will be a decrease. Therefore it appears that propagation at velocities above $1.8 \mathrm{~m} / \mathrm{s}$ cannot be due to fibre conduction, whether or not influenced by an increase in extracellular $\mathrm{K}^{+}$, but must be explained by other mechanisms.

Because propagation by low resistance gap junctions is not probable in the CA 1 area (see Introduction), non-synaptic propagation based on electrical field effects may be assumed. This propagation by ephaptic interaction depends on the influence which neurons exert on their neighbours by means of their extracellular potential field. This field has to excite inactive and marginally active neurons by depolarizing their soma membranes to at least the threshold level. Both the membrane potential of these cells and the field potential should be considered. The threshold depolarization in hippocampal pyramidal cells under normal conditions is 4-5 mV (Spencer and Kandel 1961). Under epileptic conditions the increased interstitial $\mathrm{K}^{+}$concentration will lead to a decrease of the threshold depolarization. The gradient of the field potential can be large if the neurons are densely packed and the extracellular conductivity is low, as in the pyramidal layer of the CA1 area (Taylor and Dudek 1982a, 1984a, b; Haas and Jefferys 1984; Richardson et al. 1984; Snow and Dudek 1984b; Yim et al.

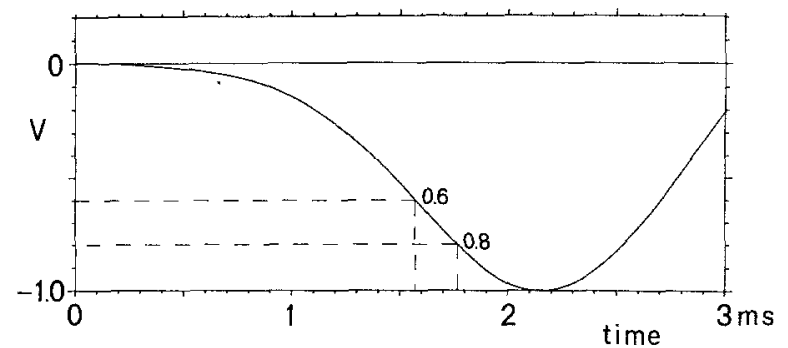

Fig. 6. The first PS of an epileptiform burst recorded in the pyramidal layer of the CAl area; maximum $\mathrm{V}=-4.18 \mathrm{mV}$

1986). It was found that the conductivity in this layer is $\approx 44 \%$ of the value in the stratum radiatum (Holsheimer 1987). Therefore, if a population of pyramidal cells generates action potentials almost simultaneously, as during epileptiform bursts, a population spike (PS) having a high amplitude may arise in the pyramidal layer. Traub et al. (1985a) concluded from a modeling study that at sufficiently high values of extracellular resistivity field effects alone can account for excitation of neighbouring neurons. Their values of simulated membrane depolarization ranged from $0.5-3.5 \mathrm{mV}$. Yim et al. (1986) recorded depolarizations of the soma membrane of pyramidal cells in CA1 with an average value of $2.7 \mathrm{mV}$, corresponding with an average of $41 \%$ of the PS amplitude in the pyramidal layer.

What will be the effect of ephaptic interaction on the velocity of propagation? The propagation velocity of a PS between the positions A and B in the pyramidal layer is estimated from the distance and the delay between the peaks of the PS in A and B. These peaks correspond to the excitation of neurons in A and B. Let us assume that the depolarization and the subsequent excitation of population $B$ is caused by the PS of population A at a small distance, of say $0.1 \mathrm{~mm}$. The delay between the PS in A and B will be influenced by several parameters: (a) the PS amplitude in A, (b) the decay of the PS amplitude from A to B, $(c)$ the delay between the PS and the resulting change in membrane potential, (d) the threshold depolarization of the pyramidal cells in B and (e) the delay between the threshold depolarization and the peak of the action potential in B. Let us further assume that a PS, originating in $\mathrm{A}$, causes a membrane depolarization of $2.5 \mathrm{mV}$ in the cells in $\mathrm{B}$ and that their excitation threshold is $2.0 \mathrm{mV}$. If the propagation velocity should be $0.33 \mathrm{~m} / \mathrm{s}$, the delay of the PS between $A$ and $B$ (distance $0.1 \mathrm{~mm}$ ) will be $0.30 \mathrm{~ms}$. If the threshold depolarization of the cells in $B$ varies (due to a change in interstitial $\mathrm{K}^{+}$concentration), the change in delay can be calculated from the shape of the PS (Fig. 6). At a threshold de- 
polarization of $1.5 \mathrm{mV}(=0.60$ of the maximum depolarization) instead of $2.0 \mathrm{mV}(=0.80)$ the cells in B will be excited $0.20 \mathrm{~ms}$ earlier by the same PS and this will result in a propagation velocity of $1.00 \mathrm{~m} / \mathrm{s}$. In this example the propagation velocity will increase by $200 \%$ due to a decrease in threshold depolarization by only $25 \%$. Thus, the velocity of ephaptic propagation will increase at an increased $\mathrm{K}^{+}$concentration. In contrast, the conduction velocity of nerve fibres may decrease (Poolos et al. 1987).

How can different propagation velocities of the PS in various parts of the CA1 area be explained? As shown before, ephaptic propagation may have different velocities in different parts of the CA1 area if the $\mathrm{K}^{+}$concentration differs slightly. Different propagation velocities of the PS may also result from fibre conduction, even at a constant conduction velocity. If the number of activated synapses at the pyramidal cells decreases, these cells will depolarize more slowly and the interval between CAP and PS will increase. However, a change in the propagation velocity of the PS was never observed in the control experiments, so that a variation in synaptic drive does not seem to be a probable explanation. Ephaptic propagation in the pyramidal layer, with a varying interstitial ionic composition, may explain the change in velocity observed in several experiments. The effect of increasing the $\mathrm{K}^{+}$concentration in the bathing medium on the threshold depolarization of the pyramidal cells was shown by an increase of the PS amplitude, which represents the number of simultaneously excited cells. However, we did not observe a change in propagation velocity when varying the $\mathrm{K}^{+}$concentration in experiments on 2 slices. We neither observed a change in propagation velocity at an increase of the $\mathrm{Ca}^{2+}$ concentration from 1 to $5 \mathrm{mM}$.

A wide range of propagation velocities has also been reported for burst activity in the hippocampal CAl area in a low-calcium medium by Haas and Jefferys (1984). They found that ephaptic propagation varied from $0.001-0.1 \mathrm{~m} / \mathrm{s}$, depending on the type of burst (with a gradual or an abrupt onset). The range of velocities is much lower than in this study, but this may be attributed to different experimental conditions.

Regarding the propagation of epileptiform activity another relevant observation in the analysis of laminar field potentials in the CA1 area during 4-AP epileptiform bursts has been made (Holsheimer et al., in preparation). The initial current sink of spontaneous bursts and bursts triggered by stimulation was at the pyramidal layer, except for stimulation of the Schaffer collaterals which was followed by an initial sink in the stratum radiatum (field EPSP). Therefore the propagation of epileptiform bursts is usually attended by a direct depolarization of the pyramidal somata, which points to ephaptic interaction or excitatory synaptic activity at or near the pyramidal layer, and not to Schaffer collateral propagation.

In contrast to the observations of Schwartzkroin and Prince (1978), Wong and Traub (1983), as well as Voskuyl and Albus (1985) we found that spontaneous epileptiform bursts are not exclusively initiated in the CA2-CA3 area, but can also start from the CA1 area. This finding was confirmed by recordings of spontaneous bursts in the CAl area after complete isolation from other parts of the hippocampal slice. This can be due to the rather thick slices $(600 \mu \mathrm{m})$, favouring excitatory interaction between pyramidal cells by fibre connections perpendicular to the lamellar plane (Christian and Dudek 1988). Hablitz (1984) also observed spontaneous bursts originating in the CAl area of slices $400-550 \mu \mathrm{m}$ thick. Although a site of seizure initiation may be created by local damage, it is not likely that this will occur on penetration of our $33 \mu \mathrm{m}$ diameter recording electrodes. Knowles et al. (1987), who used $61 \mu \mathrm{m}$ diameter electrodes in CA2-3, found that spontaneous bursts always originated at a discrete focus at or near CA2.

It can be concluded that the propagation velocity of 4-AP epileptiform bursts in the hippocampal CA1 area varies in a much wider range than the velocities of the fibre bundles in CA1. The mechanism of ephaptic interaction, which is favoured under epileptic conditions, may lead to a large variation of the propagation velocity. Therefore the propagation mechanism of epileptiform activity can be ephaptic interaction in the pyramidal layer or conduction by nerve fibres with synapses at or close to the pyramidal layer.

\begin{abstract}
Acknowledgements. We wish to thank J.H. Koolstra, A. Buyen van Weelden and $\mathrm{R}$. Boshuis for writing the computer programs, G. Heupink for his assistance in the experiments and the data processing, and A.J. Verloop for preparing the electrode arrays. We also wish to thank Dr. W.J. Wadman for his comments and criticism, Miss G.G.M. Steijlen for typing the manuscript, and $J$. Baxter for correcting the English. This work was supported by grant A-33 from CLEO (National Committee for Epilepsy Research).
\end{abstract}

\section{References}

Andersen P, Bliss TVP, Skrede KK (1971) Unit analysis of hippocampal population spikes. Exp Brain Res 13:208-221 Andersen P, Silfvenius H, Sundberg SH, Sveen O, Wigström H 
(1978) Functional characteristics of unmyelinated fibres in the hippocampal cortex. Brain Res 144:11-18

Ayala GF, Dichter M, Gumnit RJ, Matsumoto H, Spencer WA (1973) Genesis of epileptic interictal spikes: new knowledge of cortical feedback systems suggests a neurophysiological explanation of brief paroxysms. Brain Res 52:1-17

Christian EP, Dudek FE (1988) Electrophysiological evidence from glutamate microapplications for local excitatory circuits in the CA1 area of rat hippocampal slices. J Neurophysiol 59:110-123

Dichter M, Spencer WA (1969) Penicillin-induced interictal discharges from the cat hippocampus. II. Mechanisms underlying origin and restriction. J Neurophysiol 32:663-687

Fisher RS, Pedley TA, Moody WJ, Prince DA (1976) The role of extracellular potassium in hippocampal epilepsy. Arch Neurol 33:76-83

Fricke RA, Prince DA (1984) Electrophysiology of dentate gyrus granule cells. J Neurophysiol 51:195-209

Gjerstad L, Andersen P, Langmoen IA, Lundervold A, Hablitz $J$ (1981) Synaptic triggering of epileptiform discharges in CA1 pyramidal cells in vitro. Acta Physiol Scand 113:245-252

Green JD, Petsche H (1961) Hippocampal electrical activity. IV. Abnormal electrical activity. Electroenceph Clin Neurophysiol 13:868-879

Haas HL, Jefferys JGR (1984) Low-calcium field burst discharges of CA1 pyramidal neurones in rat hippocampal slices. J Physiol 354: 185-201

Hablitz JJ (1984) Picrotoxin-induced epileptiform activity in hippocampus: endogenous versus synaptic factors. J Neurophysiol 51:1011-1027

Heinemann U, Lux HD, Gutnick MJ (1977) Extracellular free calcium and potassium during paroxysmal activity in the cerebral cortex of the cat. Exp Brain Res 27:237-243

Heinemann U (1987) Basic mechanisms of the epilepsies. In: Halliday AM, Butler SR, Paul R (eds) A textbook of clinical neurophysiology. Wiley, New York, pp 497-534

Holsheimer J (1987) Electrical conductivity of the hippocampal CA1 layers and application to current-source-density analysis. Exp Brain Res 67: 402-410

Jefferys JGR (1981) Influence of electric fields on the excitability of granule cells in guinea-pig hippocampal slices. J Physiol 319: $143-152$

Jefferys JGR, Haas HL (1982) Synchronized bursting of CAl hippocampal pyramidal cells in the absence of synaptic transmission. Nature $300: 448-450$

Johnston D, Brown TH (1981) Giant synaptic potential hypothesis for epileptiform activity. Science 211:294-297

Knowles WD, Traub RD, Strowbridge BW (1987) The initiation and spread of epileptiform bursts in the in vitro hippocampal slice. Neuroscience 21:441-455

Konnerth A, Heinemann U, Yaari Y (1986) Nonsynaptic epileptogenesis in the mammalian hippocampus in vitro. I. Development of seizurelike activity in low extracellular calcium. J Neurophysiol 56:409-423

Krnjevič K, Morris ME, Reiffenstein RJ, Ropert N (1982) Depth distribution and mechanism of changes in extracellular $\mathrm{K}^{+}$and $\mathrm{Ca}^{2+}$ concentrations in the hippocampus. Can J Physiol Pharmacol 60:1658-1671

Leung L-WS (1979) Potentials evoked by alvear tract in hippocampal CA1 region of rats. I. Topographical projection, component analysis, and correlation with unit activities. J Neurophysiol 42: 1557-1570

Lorente de Nó R (1934) Studies on the structure of the cerebral cortex. II. Continuation of the study of the ammonic system. J Psychol Neurol 46:113-177
MacVicar BA, Ropert N, Krnjevič K (1982) Dye-coupling between pyramidal cells of rat hippocampus in vivo. Brain Res 238:239-244

Mody I, Lambert JDC, Heinemann U (1987) Low extracellular magnesium induces epileptiform activity and spreading depression in rat hippocampal slices. $\mathrm{J}$ Neurophysiol $57: 869-888$

Poolos NP, Mauk MD, Kocsis JD (1987) Activity-evoked increases in extracellular potassium modulate presynaptic excitability in the CA1 region of the hippocampus. J Neurophysiol 58:404 416

Prince DA (1978) Neurophysiology of epilepsy. Ann Rev Neurosci 1:395-415

Richardson TL, Turner RW, Miller JJ (1984) Extracellular fields influence transmembrane potentials and synchronization of hippocampal neuronal activity. Brain Res 294:255262

Schmalbruch H, Jahnsen H (1981) Gap junctions on CA3 pyramidal cells of guinea pig hippocampus shown by freeze-fracture. Brain Res 217:175-178

Schwartzkroin PA, Prince DA (1978) Cellular and field potential properties of epileptogenic hippocampal slices. Brain Res 147:117-130

Snow RW, Dudek FE (1984a) Synchronous epileptiform bursts without chemical transmission in CA2, CA3 and dentate areas of the hippocampus. Brain Res 298:382-385

Snow RW, Dudek FE (1984b) Electrical fields directly contribute to action potential synchronization during convulsantinduced epileptiform bursts. Brain Res 323:114-118

Somjen GG, Giacchino JL (1985) Potassium and calcium concentrations in interstitial fluid of hippocampal formation during paroxysmal responses. J Neurophysiol 53: 1098-1108

Spencer WA, Kandel ER (1961) Electrophysiology of hippocampal neurons. III. Firing level and time constant. J Neurophysiol 24:260-271

Taylor CP, Dudek FE (1982a) Synchronous neural afterdischarges in rat hippocampal slices without active chemical synapses. Science 218:810-812

Taylor CP, Dudek FE (1982b) A physiological test for electrotonic coupling between CA1 pyramidal cells in rat hippocampal slices. Brain Res 235:351-357

Taylor CP, Dudek FE (1984a) Excitation of hippocampal pyramidal cells by an electric field effect. J Neurophysiol 52:126-142

Taylor CP, Dudek FE (1984b) Synchronization without active chemical synapses during hippocampal afterdischarges. J Neurophysiol 52:143-155

Tielen AM, Lopes da Silva FH, Mollevanger WJ (1981) Differential conduction velocities in perforant path fibres in guinea pig. Exp Brain Res 42:231-233

Traub RD, Dudek FE, Taylor CP, Knowles WD (1985a) Simulation of hippocampal afterdischarges synchronized by electrical interactions. Neuroscience 14:1033-1038

Traub RD, Dudek FE, Snow RW, Knowles WD (1985b) Computer simulations indicate that electrical field effects contribute to the shape of the epileptiform field potential. Neuroscience 15:947-958

Verloop AJ, Holsheimer J (1984) A simple method for the construction of electrode arrays. J Neurosci Meth $11: 173-178$

Vliet GH van der, Holsheimer J, Bingmann D (1980) Caiculation of the conduction velocity of short nerve fibres. Med Biol Eng Comput 18:749-757

Voskuyl RA, Albus H (1985) Spontaneous epileptiform discharges in hippocampal slices induced by 4-aminopyridine. Brain Res 342:54-66 
Wong RKS and Traub RD (1983) Synchronized burst discharge in disinhibited hippocampal slice. I. Initiation in CA2-CA3 region. J Neurophysiol 49:442-458

Yaari Y, Konnerth A, Heinemann U (1983) Spontaneous epileptiform activity of CA1 hippocampal neurons in low extracellular calcium solutions. Exp Brain Res 51:153-156

Yaari Y, Konnerth A, Heinemann U (1986) Nonsynaptic epileptogenesis in the mammalian hippocampus in vitro. II. Role of extracellular potassium. J Neurophysiol $56: 424-438$
Yim CC, Krnjevič K, Dalkara T (1986) Ephaptically generated potentials in CAl neurons of rat's hippocampus in situ. J Neurophysiol 56:99-122

Received July 13, 1987; received in final form December 16, 1988/ Accepted March 16, 1989 